\title{
Desarrollo de una escala breve para medir actitudes hacia una alimentación saludable en población escolar preadolescente
}

\author{
Development of a brief scale to measure attitudes towards a \\ healthy food in a school population of pre-adolescents
}

\begin{abstract}
ASTRACT
Unhealthy eating habits are one of the main causes that contribute to non-communicable diseases (NCD). Few instruments are available to evaluate the impact of interventions as well as associated factors. Our aim was to design and evaluate a brief scale to measure attitudes towards healthy eating habits in a school population. A psychometric study was carried out. The sample (non-probability sampling) was composed of 1,238 students from public and semi-private schools in Antofagasta, Chile with ages ranging between 10 and 13, and an average age of 11.98 $(S D=1.3)$. A higher percentage of informants were female $(52.9 \%, n=655)$. Results: Factor analysis showed good indicators of adjustment in the attitude scale for healthy eating. The scale was composed of a global dimension and two specific dimensions, "attitude towards food" and "healthy behavior." We conclude that the brief survey has adequate values based on the internal structure of the test that confirm its validity.

Keywords: food; nutrition; health promotion; attitude towards health; questionnaire; risk assessment.
\end{abstract}

\section{INTRODUCCIÓN}

Las enfermedades no transmisibles (ENT) son las causantes de aproximadamente 17, 5 millones de muertes cada año'. Chile no escapa a esta tendencia mundial, dado que más de un $25 \%$ de su población presenta algunas de estas patologías ${ }^{2}$. En gran parte de las ENT encontramos factores de riesgo comunes que contribuyen a su desarrollo, entre estos la alimentación no saludable ${ }^{3}$. En Chile, uno de cada tres niños tiene un peso sobre el rango normal ${ }^{4}$, teniendo mayores probabilidades de padecer a edades más tempranas enfermedades ENT como la diabetes tipo 2, hipertensión arterial, dislipidemia y enfermedades cardiovasculares ${ }^{5,6}$, las que, al no ser tratadas a tiempo, se acrecientan paulatinamente a lo largo de la vida adulta ${ }^{7}$, convirtiéndose en uno de los principales desafíos en materia de salud pública ${ }^{8}$.

En este marco, el Ministerio de Salud de Chile (MINSAL) ha
Heleny Méndiz-Rojas ${ }^{1}$, Rodrigo Ferrer ${ }^{2}$, Alfonso Urzúa ${ }^{3}$, Ignacio Aguaded ${ }^{4}$.

1. Línea de Investigación en Educación y Comunicación, Universidad Católica del Norte, Escuela de Periodismo, Antofagasta, Chile. 2. Departamento de Filosofía y Psicología, Universidad de Tarapacá, Arica, Chile.

3. Línea de Investigación en Salud, Bienestar y Calidad de Vida, Escuela de Psicología, Universidad Católica del Norte, Antofagasta, Chile. 4. Línea de Investigación en Educación y Comunicación, Departamento de Educación, Universidad de Huelva, España.

Dirigir correspondencia a: Heleny Méndiz-Rojas, Escuela de Periodismo, Universidad Católica del Norte. Avda. Angamos 0610, Antofagasta. Fono: 56.55.23555821. Email: hmendiz@ucn.cl

Este trabajo fue recibido el 20 de enero de 2017. Aceptado con modificaciones el 24 de abril de 2017 y aceptado para ser publicado el 09 de mayo de 2017.

venido planteado la promoción de la alimentación saludable y la actividad física, entre otros factores de riesgo de las ENT, como respuestas a las necesidades de la población, implementando diversos programas de intervención en este ámbito ${ }^{9,10}$. Estas intervenciones presentan dos grandes dificultades, en primer lugar, no han logrado ser exitosas a nivel de cambios en los hábitos alimenticios, salvo algunos ensayos en pequeña escala y por un tiempo determinado, persistiendo las tasas elevadas en las diferentes edades ${ }^{11}$. En segundo lugar, existen dificultades en la medición de los efectos de las intervenciones sobre la incorporación de hábitos y conductas alimentarias saludables dado por diversos factores, tales como: a) el resultado final (conducta incorporada) se mide solo a través del auto-reporte ${ }^{12} \mathrm{O}$ reporte de los tutores ${ }^{13}$ de la ingesta, con la consecuente distorsión de la información obtenida dado por el efecto del recuerdo y la deseabilidad social, b) la dificultad de 
seguir en el tiempo la conducta /hábito "teóricamente incorporado", c) los cuestionarios existentes se centran preferentemente en la pesquisa de trastornos asociados a la conducta alimentaria ${ }^{14}$ y d) los cuestionarios suelen ser excesivamente largos y diseñados para población adulta o adolescente ${ }^{15-16}$ y no para menores en etapa de pre-adolescencia.

Con base a lo anterior, y dada la necesidad de maximizar las tasas de retorno de los cuestionarios y minimizar los tiempos de respuesta, es que el objetivo de esta investigación fue desarrollar una versión abreviada de la escala de actitud hacia la alimentación saludable ${ }^{17}$ desarrollada para población escolar de 10 a 14 años, permitiendo así reducir el error de medida de la escala original, incrementando su fiabilidad, a la vez que obtener evidencia psicométrica acorde con el actual estado del arte de la disciplina.

\section{MATERIAL Y MÉTODOS \\ Diseño y participantes}

La presente investigación corresponde a un estudio instrumental. El muestreo fue no probabilístico, constituido por un total de 1.238 estudiantes de segundo ciclo básico, $52,9 \%(n=655)$ mujeres y $47,2 \%(n=583)$ hombres, con una edad media de 11,98 años (Desviación Típica (DT)= 1,3 años) y proveniente de establecimientos educacionales de la ciudad de Antofagasta.

Instrumento

La Escala de actitud hacia la alimentación saludable (anexo 1) fue desarrollada en una investigación anterior para evaluar el impacto de capsulas radiales sobre la actitud hacia la alimentación saludable ${ }^{17}$. La versión inicial está compuesta de una dimensión global y dos dimensiones específicas denominadas "actitud frente a los alimentos" (7 ítems) y "conductas saludables" (5 ítems). Las preguntas corresponden a afirmaciones actitudinales formuladas desde el modelo tri-componental (afecto, cognición y conducta) de las actitudes ${ }^{18}$ contestadas con un formato de respuesta tipo Likert de 5 puntos ( 1 "completamente en desacuerdo" y 5 "completamente de acuerdo"). Las evidencias iniciales presentaron niveles adecuados de fiabilidad $(>0,80)^{19}$, estimados mediante Alpha de Cronbach, y evidencia de validez basada en la estructura interna del test, mediante análisis factorial exploratorio.

\section{Procedimiento}

Se invitó a participar a 6 establecimientos educacionales, de los cuales aceptaron participar 2. Primeramente, se contactó e informó a los tutores de la posible participación para responder la escala de actitud hacia la alimentación saludable. Posteriormente, se aplicó el cuestionario, durante el horario escolar, a aquellos estudiantes que, además de proporcionar su asentimiento informado (aprobado por el Comité de Ética de la Universidad Católica del Norte), retornaron con el consentimiento informado de su tutor legal para participar.

Con la información obtenida, se procedió a depurar el instrumento de medida (i.e. a partir de las saturaciones

\begin{tabular}{|c|c|c|c|c|c|}
\hline \multicolumn{6}{|c|}{$\begin{array}{l}\text { Anexo } 1 \\
\text { eactivos instrumento original y versión abreviada }\end{array}$} \\
\hline $\mathbf{N}^{\circ}$ & Reactivo & $\begin{array}{l}\text { Actitud frente a } \\
\text { los alimentos }\end{array}$ & $\begin{array}{l}\text { Conductas } \\
\text { saludables }\end{array}$ & Inverso & $\begin{array}{c}\text { Escala } \\
\text { abreviada }\end{array}$ \\
\hline 1 & Me gusta más la bebida que el agua & * & & & * \\
\hline 2 & $\begin{array}{l}\text { Me gustan los negocios de comida rápida } \\
\text { como McDonalds, Doggis u otros }\end{array}$ & * & & & * \\
\hline 3 & $\begin{array}{l}\text { Prefiero ir al patio de comidas del Mall } \\
\text { que comer en casa }\end{array}$ & * & & & * \\
\hline 4 & Tomo leche todas las mañanas & & * & * & \\
\hline 5 & La leche es sólo para los bebés & * & & & \\
\hline 6 & $\begin{array}{l}\text { Cuando llego a casa, me instalo frente a la tele } \\
\text { hasta que me llamen a comer }\end{array}$ & & * & & * \\
\hline 7 & $\begin{array}{l}\text { Cuando llego a casa, me instalo en el computador } \\
\text { hasta que me llamen a comer }\end{array}$ & * & & & \\
\hline 8 & Me gustaría hacer deporte & & * & * & * \\
\hline 9 & $\begin{array}{l}\text { Es más entretenido jugar con mis amigos que estar } \\
\text { en casa jugando computador }\end{array}$ & & * & * & * \\
\hline 10 & Prefiero ver televisión que salir a jugar con mis amigos & & * & & * \\
\hline 11 & Prefiero comer papas fritas que una fruta & * & & & \\
\hline 12 & Comer chatarra está de moda & $*$ & & & \\
\hline
\end{tabular}


factoriales), estimar fiabilidades de las dimensiones, evidencia de validez basada en la estructura interna del test, invarianza factorial por sexo, baremos.

\section{Análisis estadístico}

Se realizaron estimaciones de fiabilidad mediante Alpha de Cronbach y baremación por deciles, con la versión 21 del paquete estadístico IBM SPSS Statistics. Adicionalmente, se realizaron análisis factoriales confirmatorios, pruebas de invarianza factorial y estimaciones de fiabilidad mediante coeficientes omega con la versión 7.14 de MPLUS.

Debido a que las variables de medida son de carácter ordinal y discretas, y de acuerdo a las recomendaciones de diversos autore ${ }^{20}$, los análisis factoriales confirmatorios se realizaron a partir de la matriz de correlaciones policóricas. Además, siguiendo recomendaciones para analizar variables ordinales discretas no normales ${ }^{21}$, se utilizó el método de estimación mínimos cuadrados ponderados multivariantes (WLSMV), el cual es robusto en dichas condiciones.

\section{RESULTADOS}

En primer lugar se realizó un análisis factorial confirmatorio del modelo original (Figura 1) ${ }^{17}$, utilizando sólo la muestra del pre-test. Sin embargo, el modelo inicial presentó niveles de ajuste bajo los estándares recomendados en la literatura (i.e. $\mathrm{CFI}>0,95 ; \mathrm{TLI}>0,95 ; \mathrm{RMSEA}<0,08)^{22}$, dando cuenta que la estructura teórica impuesta no logra reproducir adecuadamente las relaciones observadas entre las variables (Tabla 1).

Debido a esto, se procedió a depurar iterativamente el modelo inicial, excluyendo los ítems número "4", "5", "7", "11" y "12", ya sea por bajos niveles de representatividad de las variables latentes $(\lambda<0,4)$, escasa variabilidad de

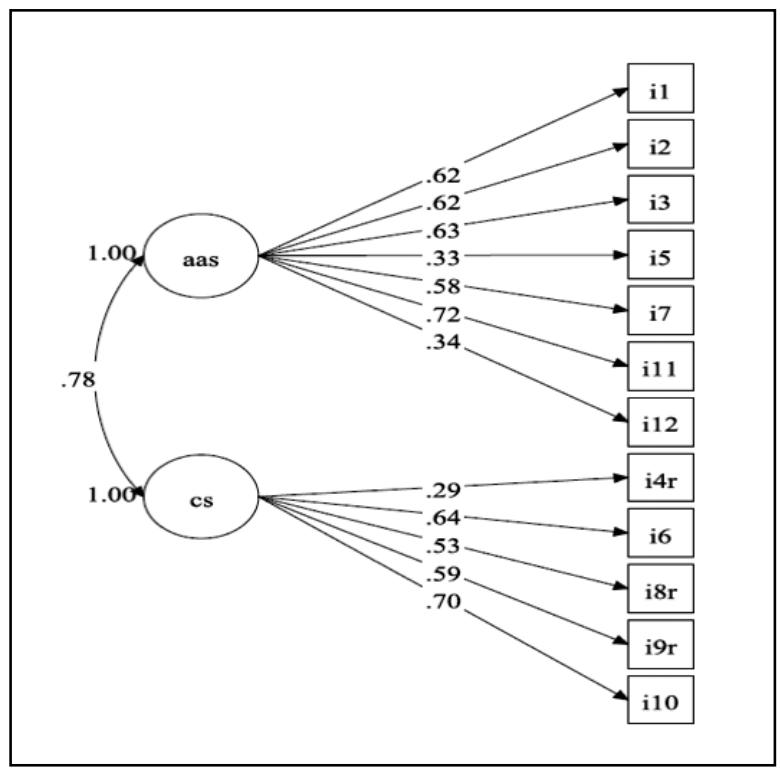

Figura 1. Modelo inicial sin depurar, con coeficientes estandarizados. las puntuaciones y/o duplicidad factorial. Finalmente el modelo final depurado (Figura 2) ofrece una versión más parsimoniosa que la inicial ( $\Delta \chi 2=-41$ ) y con niveles de ajuste adecuados (Tabla 1), sin cambios sustantivos para la interpretación de la escala. Adicionalmente, se observa que puede sostenerse la invarianza métrica del modelo $(\chi 2=4,11$; $\mathrm{DF}=5 ; \mathrm{p}=0,532)$ entre hombres y mujeres, evidenciando ser una adecuada representación de ambos grupos.

El primer factor, denominado actitud frente a la alimentación saludable, queda finalmente constituido por los reactivos: "una buena alimentación significa tener una vida sana", "la obesidad es un problema que tienen muchos niños" y "comer sano me ayuda a tener más energía para jugar y estudiar"

El segundo factor, denominado conductas saludables, incluye las siguientes afirmaciones: "la comida sana me ayuda a ser inteligente", "acompaño el almuerzo y la cena con pan", "me gusta más la bebida que el agua" y "como pescado por lo menos una vez a la semana", de las cuales dos fueron positivas y dos negativas, todas siempre relacionadas.

A partir del modelo depurado, se procedió a estimar las fiabilidades, mediante coeficiente Alpha de Cronbach y coeficiente omega por dimensión e instrumento total (Tabla 2).

De acuerdo a lo observado, tanto la dimensión general como las subdimensiones presentan niveles de fiabilidad aceptables $(\omega \geq 0,70)$.

Finalmente, se presentan los baremos en deciles de las puntuaciones totales inversas (i.e. mayor puntaje, actitud más favorable frente a los alimentos saludables o mayores conductas saludables) por dimensión y por puntaje total (Tabla 3).

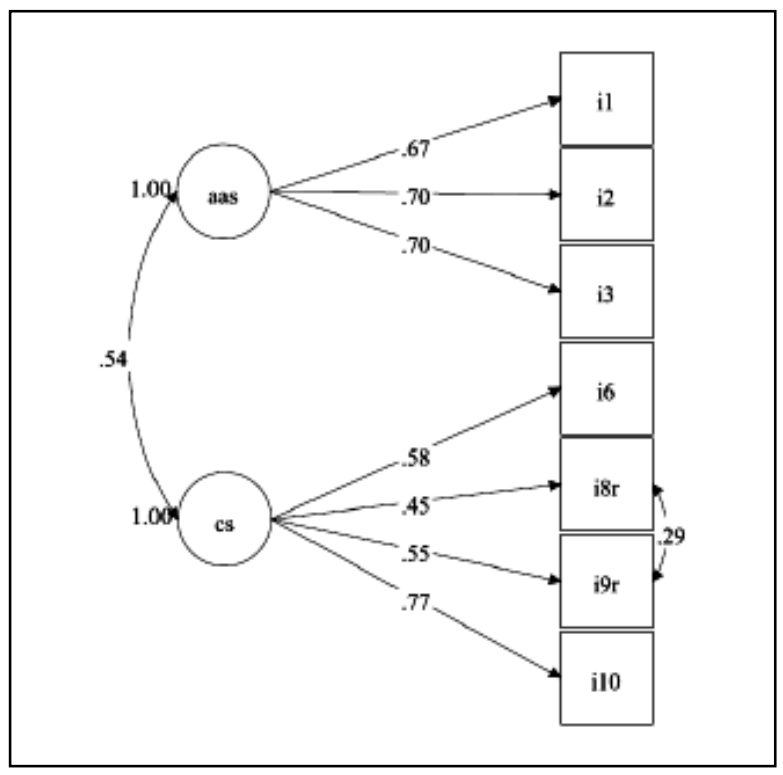

Figura 2. Modelo depurado, con coeficientes estandarizados. 


\section{TABLA 1}

Indicadores de ajuste global de los modelos

\begin{tabular}{|cccccccccccc|}
\hline & $\mathbf{N}^{\circ}$ Par & $\chi^{2}$ & DF & $\mathbf{p}$ & TLI & CFI & RMSEA & \multicolumn{2}{c|}{ RMSEA CI 90\% } & WRMR \\
\hline Figura 1 & 74 & 858,9 & 53 & 0,00 & 0,88 & 0,84 & 0,11 & 0,104 & 0,117 & 2,287 \\
Figura 2 & 44 & 105,1 & 12 & 0,00 & 0,97 & 0,95 & 0,08 & 0,066 & 0,093 & 1,150 \\
\hline
\end{tabular}

\begin{tabular}{|c|c|c|c|}
\hline & & & \\
\hline & & fiabilidad & \\
\hline & $\begin{array}{l}\text { Actitud frente a } \\
\text { los alimentos }\end{array}$ & $\begin{array}{l}\text { Conductas } \\
\text { saludables }\end{array}$ & $\begin{array}{c}\text { Conducta } \\
\text { alimentaria total }\end{array}$ \\
\hline Coeff. Alpha & 0,701 & 0,652 & 0,719 \\
\hline Coeff. Omega & 0,731 & 0,712 & 0,775 \\
\hline
\end{tabular}

\begin{tabular}{|c|c|c|c|}
\hline \multicolumn{4}{|c|}{ TABLA 3} \\
\hline \multicolumn{4}{|c|}{ Baremos en deciles calculados para la muestra total } \\
\hline Deciles & $\begin{array}{l}\text { Actitud frente a } \\
\text { los alimentos }\end{array}$ & $\begin{array}{l}\text { Conductas } \\
\text { saludables }\end{array}$ & $\begin{array}{c}\text { Conducta } \\
\text { alimentaria } \\
\text { total }\end{array}$ \\
\hline 10 & 5 & 4 & 11 \\
\hline 20 & 6 & 5 & 13 \\
\hline 30 & 8 & 6 & 14 \\
\hline 40 & 9 & 7 & 16 \\
\hline 50 & 10 & 8 & 17 \\
\hline 60 & 10 & 9 & 19 \\
\hline 70 & 11 & 10 & 20 \\
\hline 80 & 12 & 11 & 22 \\
\hline 90 & 14 & 13 & 25 \\
\hline Media & 9,31 & 8,44 & 17,75 \\
\hline DT & 3,23 & 3,46 & 5,52 \\
\hline
\end{tabular}




\section{DISCUSIÓN}

El objetivo de este reporte fue presentar las propiedades psicométricas de una versión abreviada de 7 ítems de la escala inicial de 12 desarrollada anteriormente ${ }^{17}$.

Los análisis factoriales muestran que la escala breve desarrollada producto del modelo depurado de la escala original - EBAAS, mantiene la estructura original de dos factores teóricamente vinculados, con propiedades psicométricas más robustas y adecuados niveles de fiabilidad, permitiendo recoger información tanto de la actitud frente a la alimentación saludable como de la presencia de conductas saludables, así como una medida única a partir de la combinación de ambos factores.

El primer factor, actitud frente a la alimentación saludable, apunta a un componente más cognitivo de la actitud, considerada como una tendencia psicológica expresada mediante la evaluación favorable o desfavorable hecha sobre algo o el estado de preparación o disposición mental o psicológica y neutral, que se organiza a través de la experiencia y que influye en la respuesta del sujeto ${ }^{23}$.

El segundo factor denominado conductas saludables, básicamente incluye respuestas que reflejan la conducta del ser humano en relación con su alimentación. La estructura bi-componental (actitudes y conductas) de este instrumento, responde a la necesidad de maximizar la capacidad del instrumento para detectar posibles efectos de intervenciones, ya que si bien el propósito final de las intervenciones sobre hábitos alimentarios debiese ser directamente el cambio conductual, la inclusión adicional de las actitudes tiene un doble propósito: 1) existe evidencia de que las actitudes son más sensibles al efecto que la conducta, la que suele ser más resistente al cambio ${ }^{24}$, por lo que las actitudes tienen mayor probabilidad de detectar cambios pequeños; y 2) los cambios actitudinales influencian y, en algunos casos, preceden a los cambios conductuales ${ }^{24}$, por lo que pueden detectar cambios iniciales que aún no se han manifestado conductualmente.

La importancia de la prueba está dada por su brevedad. Debemos comprender que el entorno de un niño está repleto de informaciones, novedades y estímulos. Tal vez, por eso, sea difícil para ellos mantener una atención y una concentración más allá de los 20 a 30 minutos. Con la concentración, una de las habilidades fundamentales en el proceso de conocimiento, mantenemos la atención focalizada sobre un punto de interés, durante el tiempo que sea necesario para responder el instrumento. Es indispensable tomar en cuenta que un niño no tiene el mismo tiempo de concentración que tiene un adulto. Se calcula que por cada año de vida un niño tiene entre 3 y 5 minutos de concentración. Por ende, un niño de 10 años, por ejemplo, tiene un tiempo de concentración de entre 10 y 20 minutos $^{25}$.

Una limitación de la investigación tiene que ver con la imposibilidad de contar con participantes de establecimientos privados a fin de incluir representantes de los tres tipos de establecimientos educacionales en que actualmente se encuentra dividida la educación chilena. Es necesario adicionalmente evaluar el comportamiento del instrumento en otras ciudades, ya que como se ha mencionado, este está construido y analizado en población exclusivamente de la ciudad de Antofagasta. Pese a que puede sostenerse la invarianza métrica del modelo entre hombres y mujeres, creemos necesario explorar la construcción de instrumentos con enfoque de género, toda vez que las construcciones socio-culturales asociadas al cuerpo y a la alimentación son distintas en hombres y mujeres, especialmente en una etapa de diferenciación como lo es la pre-adolescencia.

\section{CONCLUSIÓN}

La escala breve para medir actitudes hacia una alimentación saludable EBAAS, presenta una estructura bifactorial que comprende tanto conductas como actitudes hacia la alimentación saludable. Dada su brevedad, es aconsejable su uso para medir cambios de actitud en menores en edad escolar.

\section{RESUMEN}

Uno de los principales factores que contribuye al aumento de las enfermedades no transmisibles es la alimentación no saludable. Dificultades en esta área son los escasos instrumentos dedicados a evaluar tanto el impacto de las intervenciones como de los factores asociados a esta. Nuestro objetivo fue desarrollar y evaluar una escala breve para medir actitudes hacia una alimentación saludable en población escolar. Para ello, se realizó un estudio psicométrico en datos recogidos de 1.238 estudiantes con edades entre los 10 y 13 años, con un promedio de 11,98 años (DT= 1,3 años). El 52,9\% ( $n=655)$ fueron mujeres. El muestreo fue no probabilístico con participantes de establecimientos municipales y particulares subvencionados de Antofagasta. Los análisis factoriales confirmatorios presentan buenos indicadores de ajuste para una escala de actitud hacia la alimentación saludable compuesta por una dimensión global y dos dimensiones específicas denominadas "actitud frente a los alimentos" $y$ "conductas saludables". Concluimos que la escala breve desarrollada presenta adecuados valores que dan cuenta de su validez basada fundamentalmente en la estructura interna del test.

Palabras claves: alimentación; nutrición; promoción en salud; actitud en salud; cuestionario; evaluación de riesgo.

\section{BIBLIOGRAFÍA}

1. Lim SS. Vos T. Flaxman AD. Danaei G Shibuya K. AdairRohani $H$. et al. A comparative risk assessment of burden of disease and injury attributable to 67 risk factors and risk factor clusters in 21 regions, 1990-2010: a systematic analysis for the Global Burden of Disease Study 2010, Lancet 2012; 380: 2224-2260.

2. Ministerio de Salud del Gobierno de Chile -MINSAL. National Health Survey 2009-2010. Ministerio de Salud de Chile 2010. http://web.minsal.cl/portal/url/item/ bcb03d7bc28b64dfe040010165012d23.

3. Organización Mundial de la Salud. Report on the global 
situation of non-communicable diseases 2010. Organización Mundial de la Salud; 2011. http://www.who.int/nmh/ publications/ncd_report_summary_es.pdf

4. Ministerio de Salud de Chile. Basic Health Indicators, Chile 2013. Ministerio de Salud de Chile 2014. http://www.deis. cl/wp-content/uploads/2014/08/IBS-2013.pdf

5. Arnaiz P. Acevedo M. Díaz C. Bancalari R. Barja S. Aglony M. Cavada G. García H. Waist-height ratio as a predictor of cardio-metabolic risk in children and adolescents. Rev Chil Cardiol 2010; 29: 281-288.

6. Buhring K. Oliva P. Villablanca C. Rifo V. Malnutrition by excess and cardiometabolic risk in second and third grade children of Lota county, Chile. Rev Chil Nutr 2011; 38: 423428.

7. Rodríguez L. Pizarro T. Nutritional status of school children and adolescents in Chile. Rev Chil Pediatr 2006; 77: 70-80.

8. Crovetto M. Vio F. International and national background of the Chilean health promotion policy: lessons learned and future projections. Rev Chil Nutr 2009; 36: 32-45.

9. Zacarías I. Pizarro T. Rodríguez L. González D. Domper A. Programes 5 a day for the promotion of consumption of vegetables and fruits. Rev Chil Nutr 2006; 33: 276-280.

10. Biblioteca del Congreso Nacional de Chile. Law \# 20,606 On nutritional composition of food and its advertising. Biblioteca del Congreso Nacional de Chile 2015. https:// www.leychile.cl/Navegar?idNorma $=1041570$

11. Mönckeberg F. Muzzo S. The disconcerting epidemic of obesity. Rev Chil Nutr 2015; 42: 96-102.

12. Vio F. Salinas Montenegro E. González C.G. Lera L. Effect of an educational intervention on healthy eating in pre-school and school teachers and children in the Valparaíso region, Chile. Nutr Hosp 2014; 29(6): 1298-1304.

13. Lera L. Salinas J. Fretes G. Vio F. Validation of an instrument to evaluate food practices in Chilean families of schoolchildren from 4 to 7 years old. Nutr Hosp 2013; 28(6): 1961-1970.

14. Saucedo-Molina T. Unikel-Santoncini C. Validity of a multidimensional questionnaire to measure risk factors associated to eating disorders in Mexican pubescents. Rev Chil Nutr 2010; 37: 60-69.

15. Lima-Serrano M. Lima-Rodríguez J. Sáez-Bueno A. Design and validation of two scales to measure the attitude towards food and physical activity during adolescence. Rev Esp Salud Pública 2012; 86: 253-268.

16. Morales A. Montilva M. Gómez N. Cordero M. Transcultural adaptation of the scale of evaluation of eating behaviors in adolescents: "Adolescent food habits checklist". An Venez Nutr 2012; 25(1): 25-33.

17. Méndiz H. Urzúa A. Álvarez L. Arce D. Vallejos D. Zamorano A. Efficacy of radio capsules in promoting healthy eating. Rev Chi Nutr 2013; 40: 364-368.

18. Breckler SJ. Empirical validation of affect, behavior, and cognition as distinct components of attitude, J Pers Soc Psychol 1984; 47: 1191-1225.

19. Peterson RA. A meta-analysis of Cronbach's coefficient alpha. J Consumer Res 1994; 1: 381-391.

20. Garrido LE. Abad FJ. Ponsoda V. Performance of Velicer's Minimum Average Partial Factor Retention Method with Categorical Variables. Educ Psychol Meas 2011; 71: 551-570.

21. Byrne BM. Stewart SM. The MACS Approach to Testint for Multigroup Invariance of a Second-Order Structure: A walk through the process, structural equation modeling 2006; 13: 204-228.

22. Schreiber J. Nora A. Stage F. Barlow E. King J. Reporting structural equation modeling and confirmatory factor analysis results: A review. J Educ Res 2006; 99: 323-337.

23. Dreezens E. Martijn C. Tenbült P. Kok, G. Vries N. Food and values: an examination of values underlying attitudes toward genetically modified- and organically grown food products. Appetite 2005; 4: 115-122.

24. Bohner G \& Dickel N. Attitudes and attitude change. Annu Rev Psychol 2011; 62: 391-417.

25. Tokuhama-Espinosa, T. Mind, brain, and education science. A comprehensive guide to the new brain-based teaching. $W$. W. Norton \& Company, 2011. 